\title{
A cancer stem cell-like phenotype is associated with miR-10b expression in aggressive squamous cell carcinomas
}

Monika Wimmer ${ }^{1 \dagger}$, Roland Zauner ${ }^{1 \dagger}$, Michael Ablinger ${ }^{1}$, Josefina Piñón-Hofbauer ${ }^{1}$, Christina Guttmann-Gruber ${ }^{1}$, Manuela Reisenberger ${ }^{1}$, Thomas Lettner ${ }^{1}$, Norbert Niklas ${ }^{2}$, Johannes Proell ${ }^{3}$, Mila Sajinovic ${ }^{4}$, Paul De Souza ${ }^{4,5}$, Stefan Hainzl' ${ }^{1}$, Thomas Kocher ${ }^{1}$, Eva M. Murauer ${ }^{1}$, Johann W. Bauer ${ }^{1,6}$, Dirk Strunk ${ }^{7}$, Julia Reichelt ${ }^{1}$, Albert Sleiman Mellick ${ }^{4,8}$ and Verena Wally ${ }^{1 *}$

\begin{abstract}
Background: Cutaneous squamous cell carcinomas (CSCC) are the primary cause of premature deaths in patients suffering from the rare skin-fragility disorder recessive dystrophic epidermolysis bullosa (RDEB), which is in marked contrast to the rarely metastasizing nature of these carcinomas in the general population. This remarkable difference is attributed to the frequent development of chronic wounds caused by impaired skin integrity. However, the specific molecular and cellular changes to malignancy, and whether there are common players in different types of aggressive CSCCs, remain relatively undefined.
\end{abstract}

Methods: MiRNA expression profiling was performed across various cell types isolated from skin and cSCCs. Microarray results were confirmed by qPCR and by an optimized in situ hybridization protocol. Functional impact of overexpression or knock-out of a dysregulated miRNA was assessed in migration and 3D-spheroid assays. Samplematched transcriptome data was generated to support the identification of disease relevant miRNA targets.

Results: Several miRNAs were identified as dysregulated in cSCCs compared to control skin. These included the metastasis-linked miR-10b, which was significantly upregulated in primary cell cultures and in archival biopsies. At the functional level, overexpression of miR-10b conferred the stem cell-characteristic of 3D-spheroid formation capacity to keratinocytes. Analysis of miR-10b downstream effects identified a novel putative target of miR-10b, the actin- and tubulin cytoskeleton-associated protein DIAPH2.

Conclusion: The discovery that miR-10b mediates an aspect of cancer stemness - that of enhanced tumor cell adhesion, known to facilitate metastatic colonization - provides an important avenue for future development of novel therapies targeting this metastasis-linked miRNA.

Keywords: Squamous cell carcinoma, Epidermolysis bullosa, miR-10b, Metastasis, Cancer stem cell

* Correspondence: v.wally@salk.at

†Monika Wimmer and Roland Zauner contributed equally to this work. ${ }^{1}$ EB House Austria, Research Program for Molecular Therapy of Genodermatoses, Department of Dermatology \& Allergology, University Hospital of the Paracelsus Medical University, 5020 Salzburg, Austria Full list of author information is available at the end of the article

\section{Background}

While the incidence of cutaneous squamous cell carcinomas (cSCC) in Western industrialized societies has increased in recent years, it has remained relatively easy to treat, especially at early stages of the disease [1]. However, in some cases cSCCs metastasize, worsening the prognosis of patients. A special case of cSCCs occurs in patients

(c) The Author(s). 2020 Open Access This article is licensed under a Creative Commons Attribution 4.0 International License, which permits use, sharing, adaptation, distribution and reproduction in any medium or format, as long as you give appropriate credit to the original author(s) and the source, provide a link to the Creative Commons licence, and indicate if changes were made. The images or other third party material in this article are included in the article's Creative Commons licence, unless indicated otherwise in a credit line to the material. If material is not included in the article's Creative Commons licence and your intended use is not permitted by statutory regulation or exceeds the permitted use, you will need to obtain permission directly from the copyright holder. To view a copy of this licence, visit http://creativecommons.org/licenses/by/4.0/ The Creative Commons Public Domain Dedication waiver (http://creativecommons.org/publicdomain/zero/1.0/) applies to the data made available in this article, unless otherwise stated in a credit line to the data. 
suffering from the rare genodermatosis recessivedystrophic epidermolysis bullosa (RDEB). These patients are at high risk of developing a particularly aggressive type of $\mathrm{CSCC}$ with a high metastatic potential that is linked to changes in the extracellular matrix (ECM), caused by lossof-function mutations in the COL7A1 gene [2]. A lack of functional type-VII collagen (C7) at the dermal-epidermal junction (DEJ) sensitizes skin to blistering and erosions, upon minor physical stress or trauma [3]. Patients with RDEB present with congenital generalized blistering and a number of severe secondary manifestations. cSCCs arise in nearly all RDEB patients by the age of 45 , and associated metastatic disease is the primary cause of premature deaths [4]. Several mechanisms are thought to contribute to the aggressive and rapidly progressing nature of RDEB-cSCCs. In general, the skin's constant need to repair itself, coupled with the stalled inflammatory processes, and aberrant TGF- $\beta$ signaling associated with microbial challenge [5-9], are considered major risk factors. To which extent these inflammatory changes are linked to the particularly aggressive form of cSCC associated with RDEB, and if these tumors have characteristics in common with cSCCs that present with an aggressive behaviour in otherwise healthy people, remains unknown.

We focused on post-transcriptional regulatory processes in aggressive cSCCs, in particular on micro-RNAs (miRNAs). MiRNAs are short (20-25 nucleotide) RNA molecules, which are key regulators of normal cell functions. In a healthy system, miRNAs are predicted to mediate the post-transcriptional control of up to $60 \%$ of all expressed genes [10]. Their dysregulation is associated with several pathologic states, including cancer, heart disease, and obesity, and they are attributed a promising potential for therapeutic developments [11, 12]. In recent years, both, oncogenic miRNAs (onco-miRs) and tumor suppressive miRNAs, have been identified as playing important roles in cancer progression. In addition, a class of miRNAs have been shown to have specific pro-metastatic properties. A key metasta-miR, miR-10b, has been associated with tumor promoting properties, as well as the growth of metastatic foci in breast cancer in various landmark studies [13-15]. MiR-10b is encoded by a highly conserved genomic region, which is located near the homeobox $\mathrm{D}(H O X D)$ cluster on chromosome 2 [16]. It has been linked to a range of functions, including regulation of angiogenesis and promotion of cell invasion [17-20]. Increased serum levels of miR$10 \mathrm{~b}$ are associated with poor prognosis in melanoma and breast cancer [21, 22], and intravenous injections of miR-10b inhibitors in tumor-bearing mice dramatically reduced breast cancer cell metastasis [13, 15, 23]. In addition, a metaanalysis of miR-10b levels and clinical outcomes in various cancers, demonstrated that overexpression was associated with poor overall survival, indicating that miR-10b might be a promising prognostic biomarker [24].
In this study, we substantially add to our understanding of the role of miR-10b, by reporting for the first time on the dysregulation of this miRNA in aggressive cSCC. Both, RDEB- and otherwise healthy donor-related cSCCs (HC-cSCCs) were classified and included in the study according to their potential to metastasize. We show that miR-10b expression is linked to a cancer stem celllike phenotype in a 3D organotypic model. Taken as a whole, this work provides a new explanation of maligancy in CSCCs, and a novel target for further development of markers and therapies to treat cSCCs.

\section{Methods}

\section{Patient samples and cell lines}

For this study, primary cells were used for microarray experiments, as well as for validations. For miR-10b overexpression, we used HPV16 E6/E7 immortalized keratinocytes. All cells were cultured in defined, serum-free CnT-Prime Epithelial Culture Medium (CELLnTEC, Cat No. CnT-PR) at $37^{\circ} \mathrm{C} / 5 \% \mathrm{CO}_{2}$ in a humidified incubator. For more detailed information regarding origin of the cell lines and donor description see Supplementary Information and Supplementary Table S1 in Additional File 1.

\section{Microarrays}

For total RNA and miRNA isolation from cell cultures, we used miRNeasy Mini Kit (Qiagen, 217004), according to manufacturer's protocol. For transcriptome analysis, an Affymetrix Clariom ${ }^{\mathrm{Tm}} \mathrm{D}$ (Thermo Fisher, 902922) was applied and an Affymetrix GeneChip ${ }^{\text {Tax }}$ miRNA 4.1 platform was used for miRNA analysis (Thermo Fisher, 902409). Arrays were performed by the service provider "Core Facility Genomics at the Medical University Vienna" in accordance with manufacturer's instructions. RNA quality was confirmed on a Bioanalyzer prior to hybridization onto a respective array. Quality assessment of microarray data was conducted via "Transcriptome Analysis Console" (Applied Biosystems v4.0.0.25).

\section{Bioinformatic data processing and statistical analysis}

All data processing and analysis was documented and performed in statistical software R (v3.5.1). Scripts are available upon request. For detailed statistical analysis refer to Supplementary Information in Additional File 1.

For statistical analysis Student's t-test was performed using the GraphPad Prism (v 5.03) or Excel software, and error bars represent standard error of mean (SEM), unless mentioned otherwise. Details regarding number of replicates can be found in figure legends.

\section{TaqMan qPCR miRNA assays}

For primary (pri)-miRNA assays cDNA was synthesized from $1 \mu \mathrm{g}$ total RNA. For reverse transcription, the High Capacity RNA-to-cDNA kit (Applied Biosystems, 
4387406) was used according to the manufacturer's protocol. TaqMan pri-miRNA assays (Thermo Fisher Scientific, 4427012, Hs03302884_pri) including the TaqMan Gene Expression Master Mix, were used. RNaseP (Thermo Fisher Scientific, 4401631) served as endogenous control.

For mature miRNA validations we used TaqMan Advanced miRNA assays (Thermo Fisher Scientific, A25576), $2 x$ Fast Advanced Master Mix, and respectively diluted miRNA templates. miR-320a-3p (Thermo Fisher Scientific, 478594_mir) was used as endogenous control. All reactions were performed in technical triplicates, and repeated as independently performed experiments for at least three times.

\section{Fluorescence-based in situ hybridization of miR-10b on cultured cells and formalin-fixed paraffin embedded (FFPE) tissue sections}

An optimized in situ hybridization protocol was applied, based on the method described by Gasch et al. [13] and the manufacturer's recommendations (Qiagen, 339450). In brief, $8 \mu \mathrm{m}$ FFPE tissue sections were deparaffinized in xylene, washed and rehydrated in serial dilution of ethanol / $\mathrm{dH}_{2} \mathrm{O}$. Antigen retrieval was performed in citrate buffer $\mathrm{pH} 6.0$ at $100^{\circ} \mathrm{C}$.

Cells were cultured in 8-well glass chamber slides (Millicell EZ Slide, PEZGS0816) and fixed with 4\% PFA in PBS at room temperature, and permeabilized by incubation in $1 \%$ Triton-X100 in PBS. After re-fixation in 4\% PFA in PBS, cells were washed twice in PBS and incubated for 20 min at $54{ }^{\circ} \mathrm{C}\left(60 \mathrm{~min}\right.$ at $52^{\circ} \mathrm{C}$ for tissue sections) in miRCURY locked-nucleid acid (LNA) miRNA ISH buffer (Qiagen, 339450). Digoxigenin labeled miRCURY LNA probes (HSA-MIR-10B-5P Cat No. 339111YD00610135BCE, SCRAMBLE-MIR Cat No. 339111YD00699004BCE, miRCURY LNA U6 Cat No. 339111YD00699002BCE, Qiagen) were diluted in hybridiziation buffer (cells: $80 \mathrm{nM}$, tissue sections: $100 \mathrm{nM}$ ) after a denaturation step at $90^{\circ} \mathrm{C}$ and incubated on cells for $30 \mathrm{~min}(60 \mathrm{~min}$ tissue sections) at $54{ }^{\circ} \mathrm{C}\left(52^{\circ} \mathrm{C}\right.$ tissue sections). After probe hybridization increasingly stringent washing steps were applied in saline-sodium citrate (SSC) buffer (Sigma, SSC 20x buffer concentrate S6639-1 L) at various concentrations and temperatures. Tris based $(100 \mathrm{mM})$ buffer was supplemented with $3 \%(\mathrm{v} / \mathrm{v})$ FCS, $1 \%(\mathrm{w} / \mathrm{v})$ hyridization blocking reagent (Roche, 339450), 0.3\% (v/v) Triton-X100 and $\mathrm{NaCl}(150 \mathrm{mM})$. Anti-DIG Fabs (Roche, 11207741910), Alexa Fluor 647 Mouse Anti-Human Cytokeratin 14, 15, 16 and 19 Clone KA4 (Supplementary Table S2 in Additional File 1) and DAPI were used for visualization and co-staining of cells and tissue sections, respectively. Slides were mounted using Prolong Gold Antifade mounting medium (Sigma, P36930).

Imaging was performed on a LSM800 Airyscan confocal microscope (Zeiss). Analysis was conducted in ImageJ (v1.52i) and CellProfiler (v3.0.0) with all steps documented in scripts and workflow files, available upon request. Cellular boundaries were detected based on respective fluorescence signals and signal intensities were integrated at single cell resolution. To confine the analysis to cytoplasmic signals, nuclei were demarked via DAPI staining and co-localizing fluorescence signals were subtracted from total fluorescence. Downstream statistical analysis was performed in statistical software R (v3.5.1).

\section{Histological analysis of tissue sections and immunofluorescence microscopy}

cSCC samples from patients undergoing tumor resection were FFPE processed [25]. Hematoxilin \& eosin (H\&E) stainings were performed for histological assessment by a dermato-histopathologist (Department of Dermatology, University Hospital Salzburg).

For immunohistochemistry (IHC), sections were incubated at $58{ }^{\circ} \mathrm{C}$ for $1 \mathrm{~h}$, and subsequently deparaffinized three times in Roti-Histol (Roth, 6640) at room temperature (RT). Next, sections were washed in decreasing alcohol concentrations $(3 \times 100 \% \mathrm{EtOH}, 2 \times 90 \%$ isopropanol, $1 \times 70 \%$ isopropanol, $1 \times \mathrm{dH}_{2} \mathrm{O}$ ). Heat-induced antigen retrieval was performed in citrate buffer $\mathrm{pH}$ 6.0. Upon blocking with $5 \%$ bovine serum albumin (BSA, Sigma, A3294) in PBS, first antibodies were diluted in $2 \%$ BSA/PBS. For all antibodies, a respective second-step control was included. Secondary antibodies were used at a 1: 500 dilution (Supplementary Table S2 in Additional File 1). DAPI was used for nuclear staining (Sigma Aldrich, D9542). The same protocol was used for cultured cells. There, about $3 \times 10^{4}$ cells were seeded into $1 \mathrm{~cm}^{2}$ chamber slides. After $24 \mathrm{~h}$ (hrs) cells were fixed for $10 \mathrm{~min}$ in $4 \%$ formaldehyde in PBS, washed once with PBS and blocked at room temperature for 1 hour with 2\% BSA in PBS. Imaging was performed on a Zeiss LSM710 confocal microscope.

\section{Cloning of MIR10B and stable transduction}

For stable expression of miR-10b in E6/E7 immortalized RDEB-KCs, the human MIR10B gene was cloned into the pMX-IRES-Blasticidin vector (Cell Biolabs Inc., RTV-016), downstream of the constitutive Pol-III U6 promoter. Primer sequences are given in Supplementary Table S3 in Additional File 1. All constructs were analyzed using Sanger sequencing before viral packaging.

Viral particle production using pMX_U6_miR10b was done as described previously [26]. Expression and maturation of miR-10b was confirmed by TaqMan qPCR (Supplementary Fig. S1C-E in Additional File 1).

\section{CRISPR-mediated knock-out of MIR10B}

Two single guide (g) RNAs, flanking the MIR10B stem loop region on chromosome 2 , were rationally designed and selected to specifically knock-out MIR1OB. Single gRNAs were synthesized and purified using the Precision 
gRNA Synthesis Kit according to the manufacturer's instructions (Thermo Fisher Scientific, A29377). Two primer sets were used for gRNA synthesis (Supplementary Table S3 in Additional File 1). Recombinant spCas9 was purchased from Polyplus-transfection (Polyplus-transfection). Ribonucleoproteins (RNPs) were complexed in a 4: 1 ratio $(3 \mu \mathrm{g}$ spCas9 and $750 \mathrm{ng}$ sgRNAs; $375 \mathrm{ng}$ each sgRNA) for $10 \mathrm{~min}$ at RT and delivered into RDEBSCC1 keratinocytes by nucleofection using the Neon Transfection System $10 \mu \mathrm{L}$ Kit (Thermo Fisher Scientific, MPK10025). SCC cells were trypsinized and washed with PBS, and $3 \times 10^{5}$ keratinocytes were resuspended in $12 \mu \mathrm{L}$ Resuspension Buffer R per reaction. RNP complexes were added to each sample and electroporated into SCC cells $\left(\sim 2.5 \times 10^{5}\right.$ cells $)$ under the following conditions: $1400 \mathrm{~V}, 20 \mathrm{~ms}, 2$ pulses. After electroporation, cells were seeded into 6-well plates containing pre-warmed antibiotic-free CnT-Prime Epithelium Culture Medium (CELLnTEC).

\section{Migration assay}

Cell motility assays were performed using 2-well silicone inserts with a defined cell-free gap, suitable for wound healing / migration assays (IBIDI, 80241). $7 \times 10^{4}$ cells in $70 \mu \mathrm{L}$ CnT-Prime medium were seeded per well. Twenty-fourh post seeding silicone inserts were removed and migration of cells was monitored at different time points by measuring cell confluence in a predefined, constant area including the gap using a Spark ${ }^{\circ}$ $10 \mathrm{M}$ multimode microplate reader imaging module (Tecan). Gap-area was calculated using ImageJ (v1.52i). In parallel to every migration assay, proliferation of each cell type was measured in 24-well plates, to exclude varying proliferation rates as confounding factor.

\section{Generation of 3D tumor spheroids}

Spheroids were grown as floating spheres in AggreWell ${ }^{\mathrm{Tx}}$ 400Ex Plates (Stem-cell Technologies, 34425) according to the manufacturer's recommendations. $2.35 \times 10^{5}$ cells / well were seeded in CnT-prime medium supplemented with $1.2 \mathrm{mM} \mathrm{CaCl}_{2}$. Spheroids were grown for $96 \mathrm{~h}$. Aggregates were transferred into a 24-well plate for imaging on a Spark ${ }^{\circ} 10 \mathrm{M}$ multimode microplate-reader with integrated imaging module (Tecan). For live/dead stainings, aggregates were purified using Corning ${ }^{\circ} \mathrm{Co}-$ star ${ }^{\circ}$ Spin- $\mathrm{X}^{\bullet}$ Centrifuge tube filters $(0.22 \mu \mathrm{m})$ (Corning, CLS8161-100EA). Viability was tested via live/dead viability/cytotoxicity kit (Thermo Fisher, L3224). Imaging was performed on a Zeiss LSM710 confocal microscope. TECAN whole well images were processed in ImageJ (v1.52i) software. All image processing was documented in ImageJ macros and downstream statistical analysis in $\mathrm{R}$ (v3.5.1) scripts, which are available upon request.
For outgrowth experiments, spheroids were transferred to standard 24-well plates and incubated at $37^{\circ} \mathrm{C}$ in a humidified incubator using CnT-Prime Epithelium Culture Medium. Cellular outgrowth was imaged $24 \mathrm{~h}$ post transfer.

\section{Semi-quantitative real-time PCR}

SqRT-PCR was performed using the $\mathrm{GoTaq}^{\circ} \mathrm{qPCR}$ Master Mix (Promega, TM318), according to the manufacturer's protocol. Glycerinaldehyd-3-phosphatdehydrogenase (GAPDH) and tubulin alpha 1 (TUBA1) were used as reference genes. For primers see Supplementary Table S3 in Additional File 1. Data collection was performed on a CFX96 (BioRad) instrument. Relative target gene expression quantities and significance levels (unpaired two-sided t-test) were calculated using the $\Delta \Delta \mathrm{Cq}$ method. All reactions were performed in technical duplicates, and performed in at least three independent experiments.

\section{Western blot analysis}

Protein extracts were generated from $\sim 5 \times 10^{5}$ cells at a confluency of $80 \%$, dissolved and homogenized in RIPA buffer (Santa Cruz, sc-24948) supplemented with 5\% B-Mercaptoethanol ( $(\mathrm{B}-\mathrm{ME})$. Proteins were separated on a $4-15 \%$ bis-tris gel (Invitrogen, Nupage, NP0323) in MOPS buffer and blotted onto a $0.45 \mu \mathrm{m}$ nitrocellulose membrane (Amersham Biosciences, Hybond-ECL, Merck, GERPN303D). Ponceau Red staining was performed for total protein analysis of ChemiDoc (BioRad) images in ImageLab software (BioRad, v5.2.1). Membranes were blocked using 5\% milk powder in TBS / 0.1\% Tween20 (Merck, 655205-250ML). All antibodies are listed in Supplementary Table S2 in Additional File 1. Membranes were washed five times using TBS / $0.1 \%$ Tween-20. HRP activity was visualized using the Amersham ECL Select Western blot detection reagent (Amersham Biosciences, RPN2235), and the ChemiDoc system (BioRad). Specificity of the DIAPH2 antibody was confirmed in DIAPH2 knock-out cells. Dosimetric semiquantitative analysis was performed in either ImageLab (BioRad, v5.2.1) or ImageJ (v 1.52i).

\section{MiR-10b mimic transfections}

Primary healthy control keratinocytes (HC-KC) were transiently transfected using Xfect transfection reagent (TaKaRa Bio, 631450/631317) with $50 \mathrm{nM}$ miR-10b-5p mimic $/ \sim 10^{5}$ cells (Thermo Fisher, miRVana, 4464066) or scrambled control (Merck, HMC0002) according to manufacturer's protocol. Cells were lysed $72 \mathrm{~h}$ posttransfection in RIPA buffer (Santa Cruz, sc-24948) supplemented with 5\% ß-ME for subsequent Western blot analysis. 


\section{Results \\ MiRNome profiling identifies up-regulation of miR-10b in RDEB-CSCC}

In order to identify differences in the miRNA expression profile between cultured primary keratinocytes (KC) and cSCC cells (Supplementary Information and Supplementary Table S1 in Additional File 1), we conducted an Affymetrix GeneChip ${ }^{\text {tm }}$ miRNA 4.1 expression analysis. Out of 2578 analyzed mature miRNAs unique to human, 50 miRNAs were found to be significantly ( $p$-value $\leq 0.05$ and false discovery rate $($ FDR $) \leq 0.2) \geq 2$-fold up- or down-regulated in RDEB-cSCCs $(n=4)$ compared to RDEB-KC $(n=6)$, and 56 in HC-cSCCs $(n=3)$ compared to healthy control (HC)-KCs $(n=5$, Supplementary Fig. S1A, Supplementary Tables S4 and S5 in Additional File 1). When analyzing miRTarbase v6.1 predicted targets of the deregulated ( $\geq 2$-fold, p-value $\leq 0.05$ and FDR $\leq 0.2)$ miRNome, various cancer-related Kyoto Encyclopedia of Genes and Genomes (KEGG) pathways, e.g. signaling pathways regulating pluripotency of stem cells, appeared significantly enriched (Supplementary Fig. S1B, Additional File 1).

To assess the ability of the miRNA expression profile to distinguish cSCCs from other experimental groups we performed principal component analysis (PCA). Annotation of the samples within the distinct clusters showed a clear separation of experimental groups (Fig. 1a). Even though the miR-10 family was found to be overall upregulated in cSCCs, in-depth analysis of major contributors driving the unsupervised cluster separation highlighted miR-10b, which was 2.2 -fold $(p<0.05)$ upregulated in RDEB-cSCCs and 2.2-fold $(p<0.05)$ in HC-cSCCs, respectively, compared to their non-malignant controls (Fig. 1b, c). Despite the fact that miR-10a appeared to be the most deregulated miRNA in RDEB-cSCCs in microarray analysis, but not in subsequent qPCR, we assume that high microarray scores most likely derived from a certain hybridization error rate, as miRs-10a and $-10 \mathrm{~b}$ differ in only one nucleotide (Fig. 1e, Supplementary Fig. S1C-E, Additional File 1). In addition, consistently increased miR-10b (on average 9-fold), but not miR-10a levels in RDEB-cSCC were found in previously generated RNA sequencing (RNA-seq) data, where immortalized $\mathrm{HC}-\mathrm{KC}$ lines were used as controls (Supplementary Fig. S1F,G, Additional File 1). Thus, we focused on miR-10b and dropped miR-10a from further experiments. Predicted miR-10b targets were further tested for gene set enrichment in cancer hallmarks (Molecular Signature Database v6.2), and showed significant association with metastatic processes like epithelial to mesenchymal transition (EMT) (Fig. 1d).

In summary, the results of a microarray based miRNA expression profiling revealed a deregulated miRNome able to distinguish cSCC from keratinocytes. In particular, miR-10b was significantly upregulated in cultured cSCC.

\section{Expression of miR-10b in CSCC tissues}

In order to localize and further substantiate upregulation of miR-10b expression in tissue, we optimized a combined IHC-ISH protocol using LNA probes.

When tissue specific expression was examined in FFPEsections of archival skin and tumor biopsies, miR-10b was found to be upregulated in CSCC, particularly in RDEBcSCCs. Expression was predominantly co-localized to keratin-positive cells (Fig. 1f, Supplementary Fig. S2A-C, Additional File 1), tumor vasculature and lymphocytes (Supplementary Fig. S2D in Additional File 1). Notably, both vasculature and hemopoietic cells have previously been shown to express miR-10b in a tumor context [15, 18, 27-29]. Further analysis of RDEB-tissues showed a strong expression of transcription factor TWIST1, a known upstream driver of miR-10b-mediated tumor malignancy. In addition, TWIST1 was also found to be upregulated in cultured SCC cells in IF and Western blot analysis (Supplementary Fig. S2E,F, Additional File 1) $[14,30]$. Single cells in an RDEB-cSCC lymphnode metastasis (RDEB-SCC LM) expressed high levels of miR-10b. Less pronounced miR-10b abundance was observed in $\mathrm{HC}$-cSCC (Fig. 1f).

To investigate whether miR-10b expression was correlated to differentiation, cells were incubated in the presence of calcium and serum to induce differentiation, and respective marker gene expression was analyzed in parallel to miR-10b expression levels. However, no correlation between differentiation and miR-10b expression was observed (data not shown).

Taken together, results of the IHC-ISH show high abundance of miR-10b in cSCC tumor biopsies.

\section{MiR-10b confers anchorage-independent aggregation capabilities to keratinocytes and attenuates mobility}

Metastasis requires the dissemination and successful establishment of clones with tumor-initiating potential at distant niches [31]. In order to investigate the biological role of miR-10b, we analyzed its expression levels in experimental cell lines using IHC-ISH, in parallel to 3D tumor spheroid formation assays. As the cytoplasm is considered as the site of miRNA maturation and action, fluorescence intensity, excluding the nuclear region, was assessed at a single cell resolution. MiR-10b was confirmed to be highly expressed in RDEB-cSCC cell lines using ISH probes specific for mature miR-10b, and in two out of three HC-cSCC cultures, as compared to primary HC-KCs (Fig. 2a, b, Supplementary Fig. S3,4 in Additional File 1).

Next, we performed aggregate formation assays to explore the capacity of experimental cell lines to form 

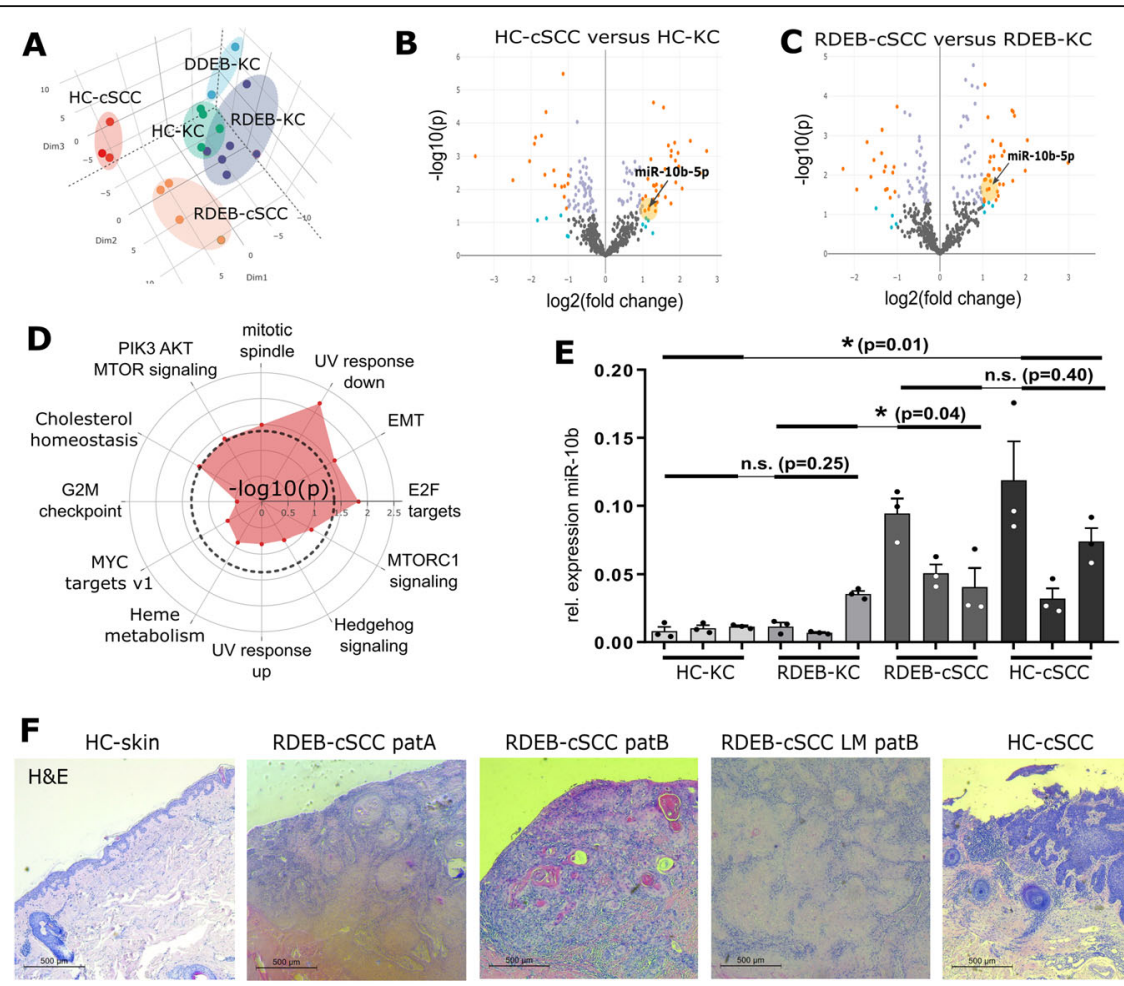

RDEB-cSCC patA
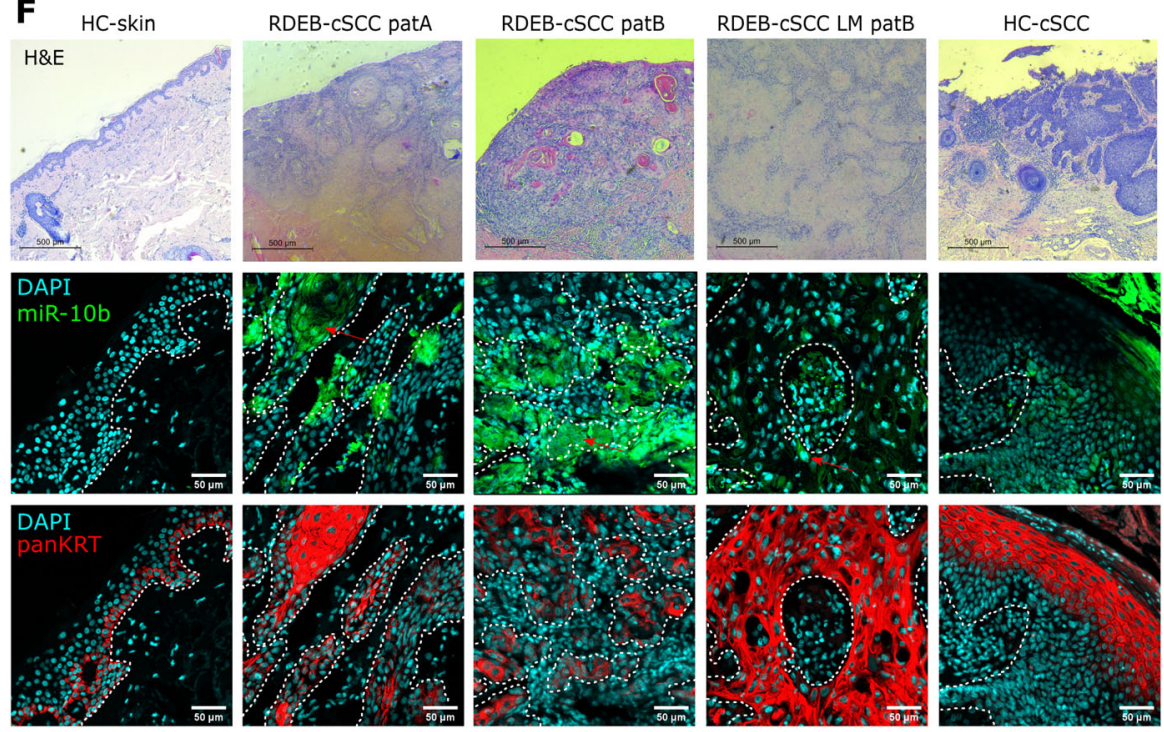

Fig. 1 miR-10b is upregulated in RDEB-CSCC. a Unsupervised clustering via PCA on miRNA expression levels clearly separated RDEB-cSCC ( $n=4$ ) and HC-CSCC $(n=3)$ samples from RDEB-KC $(n=6)$ and HC-KC $(n=5)$. Target analysis of miR-10b, which was significantly $(p \leq 0.05)$ up-regulated ( $\geq 2$-fold) in both, b HC-cSCCs and (c) RDEB-CSCCS, indicated an enrichment of pathways related to aggressive tumor phenotypes like epithelialto-mesenchymal transition (EMT) (d). e TaqMan qPCR ( $n=3$ ind. repl., mean \pm SEM, ${ }^{*} p<0.05$, n.s. non-significant, unpaired t-test), as well as ( $\mathbf{f}$ ) fluorescence-based in situ hybridization (FISH) with DIG labelled miR-10b probes (green) on FFPE tissues sections of confirmed (H\&E staining, top row) carcinomas also showed miR-10b dysregulation. White dashed line: tumor boundaries marked by pan-keratin staining (red). Scale bars: $100 \mu \mathrm{m}$ (white), 500 um (black). Sample codes (e) from left to right HC-KC: 1090KC, SKC013, SKC018; RDEB-KC: RDEB-55KC, RDEB-43KC, RDEB-29KC; RDEB-CSCC: RDEB-SCC1, RDEB-SCC2, RDEB-SCC62; HC-CSCC: SCC13, WT18SCC, A431

anchorage independent spheroids [32]. All three RDEBcSCCs, and two out of three $\mathrm{HC}$-cSCCs formed stable aggregates (Fig. 2c, d). RDEB-KCs, which were used as surrogate for functional experiments in all downstream experiments, stably overexpressing miR-10b $\left(\right.$ RDEB-KC ${ }^{\text {miR-10b }}$ ), as well as HC-KC ${ }^{\text {miR-10b }}$, phenocopied RDEB-cSCCs in the formation of stable spheroids (Fig. 2e). Expression and maturation of miR-10b, driven by the constitutive Pol-III U6 promoter, was confirmed by qPCR (Supplementary Fig.
S5A, Additional File 1). In addition, we found that $\mathrm{KC}^{\text {miR-10b }}$ were significantly smaller than their parental cells $\left(p\right.$-value $\mathrm{eDEB}^{\mathrm{RDC}}=0.002 ; p$-value $\mathrm{HC}^{\mathrm{HC}-\mathrm{KC}}<0.001$; Supplementary Fig. S6A in Additional File 1). Like in RDEB-cSCC derived aggregates, viable cells were found predominantly in the outer spheroid layers of $\mathrm{KC}^{\text {miR-10b }}$ spheres (Supplementary Fig. S5I in Additional File 1). Parental RDEB- and HC-KCs formed fewer and rather loose aggregates, which did not withstand mild pipetting (Fig. 2e). 

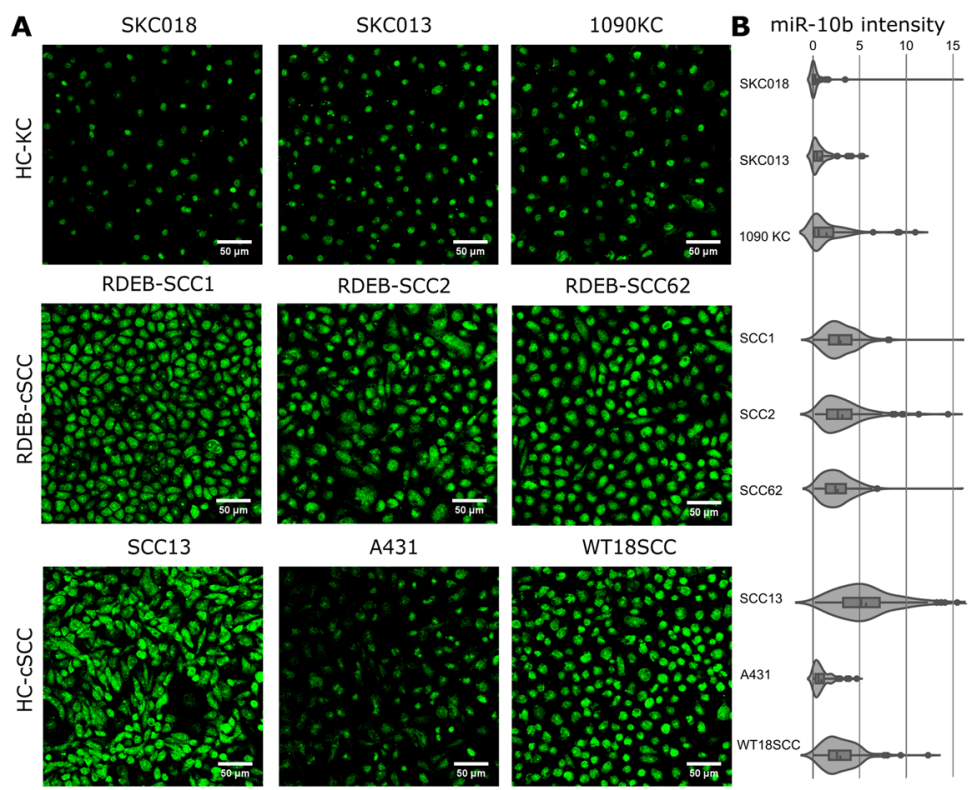

RDEB-SCC2

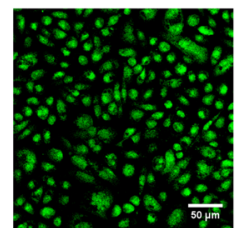

RDEB-SCC62

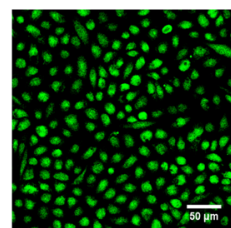

A431
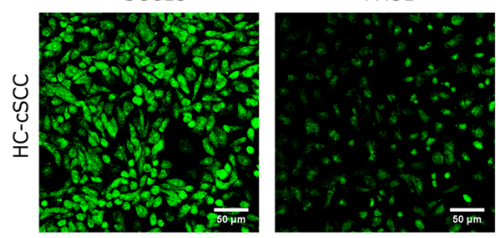

WT18SCC
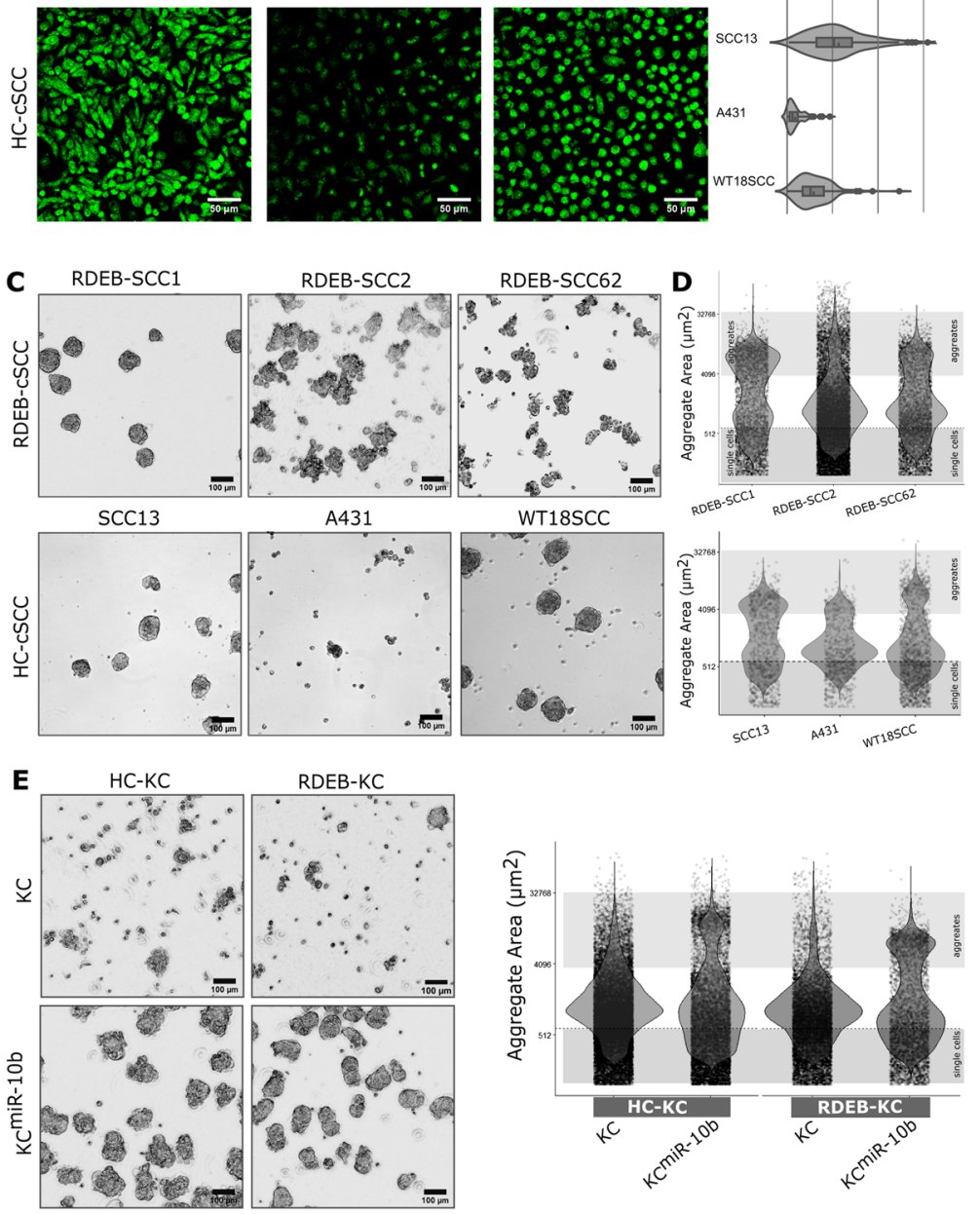

Fig. 2 Stable aggregate formation is associated with miR-10b expression. a DIG-labelled miR-10b-5p specific LNA probes (green) were used in an in-situ hybridization protocol to quantify relative expression of miR-10b in cultured cells by FITC labeled anti-DIG-Fab immunofluorescence imaging. All three tested RDEB-CSCCS and two out of three HC-CSCC cell lines demonstrated increased miR-10b signals compared to primary HCKCs. b To quantify the miR-10b expression at single cell resolution, cytoplasmic FITC signals of cells were cumulated and plotted as relative fluorescence intensity per single cell on the x-axis. Graphic shows distribution density function of miR-10b intensity of all analyzed single cells per sample. Respective negative- (scrambled) and positive- (U6) probes were used as controls (Supplementary Figs. S3 and S4 in Additional File 1). c All RDEB-CSCC lines and two out of three HC-cSCC lines demonstrated stable aggregate formation $96 \mathrm{~h}$ post seeding (representative images of aggregates). $\mathbf{d}$ Cross section of objects evaluated after transferring aggregates $\left(n \sim 7.10^{3}\right)$ into cell culture plates. Each dot represents one object. $\mathbf{e}$ In addition, overexpression of miR-10b in KCs conferred spheroid formation capacities. Sample codes HC-KC: 1090KC, RDEB-KC: RDEB-57KC. Scale bars: $50 \mu \mathrm{m}$ (white), $100 \mu \mathrm{m}$ (black) 
To further substantiate the link between miR-10b overexpression and enhanced spheroid formation capacities, we knocked-out the MIR10B gene locus in RDEBcSCC cells (RDEB-SCC1 ${ }^{\text {MIRIOB-/-}}$ ) using the CRISPR/ Cas9 technology, and performed minimal dilution to potentially obtain single clones, which was confirmed by Sanger sequencing and qPCR on mature miR-10b expression levels (Supplementary Fig. S5J,K). In spheroid formation assays we observed, that MIR10B knock-out reduced the stability of aggregates and resulted in an increased number of single cells and fragmented aggregates (Fig. 3a-c). While PCR-mediated confirmation of MIR1OB knock-out showed only bands corresponding to successful deletion, we found that over time single cells that had escaped knock-out and subsequent clearance by minimal dilution returned to dominance, independent of a potential proliferative advantage (Fig. 3d, e). This was observed in several clones and over several cultivation passages, pointing towards a potential survival advantage of cells expressing miR-10b. When subjecting these mixed cultures again to 3D-sphere formation assays, their behavior resembled that of parental cells (Fig. 3ac). Another striking difference between parental and MIR1OB-/- cells was a reduced capacity to grow out of tumor spheroids upon transfer to culture dishes. Spheroids adhered to dishes, and circularly outgrowing cells became visible after $24 \mathrm{~h}$ in RDEB-SCC1 derived aggregates, and to a much lower extent in RDEB-SCC1 ${ }^{\text {MIR } 10 B}$ ${ }_{-/-}$cells. Again, this was reversed in mixed culture experiments. A similar outgrowth pattern to RDEB-SCC1 was also observed in two out of three $\mathrm{HC}$-cSCC derived spheroid experiments (Fig. 3f).

As spheroid formation is a key attribute of cancer stem cells (CSCs), and points towards the presence of cancer stem cell-like properties, we analyzed accepted CSC markers (CD44 / CD24) [33]. RDEB-cSCC cultures showed an overall high expression of CD44, and varying levels of CD24, including a CD $44^{\text {high }} / \mathrm{CD} 24^{-/ \text {low }}$ cell population. In support of the hypothesis that miR-10b expression was associated with a CSC-like phenotype, overexpression of miR-10b in KCs resulted in an overall $\sim 2$-fold enrichment of CD44 $4^{\text {high }} / \mathrm{CD} 24^{- \text {/low }}$ cells (Supplementary Fig. S6B,C in Additional File 1).

We next examined whether the above observed properties were attributed to mobility-, or adhesion-associated mechanisms [32]. Therefore, we conducted wound closure assays using RDEB-KC ${ }^{\text {miR-10b }}$ and RDEB-SCC1 ${ }^{\text {MIRIOB-/- }}$ cells. We found that those cells expressing high levels of miR-10b (i.e. RDEB-KC ${ }^{\text {miR-10b }}$ versus parental, parental versus RDEB-SCC1 ${ }^{\text {MIR } 10 B-/-}$ ) demonstrated significantly impaired mobilization (time point $6 \mathrm{~h}: p<0.01$ ) (Fig. 4a, b). In addition, when transiently transfecting $\mathrm{SCC}^{\mathrm{MIR} 10 \mathrm{O}-/-}$ with a miR-10b mimic, as well as when using the mixed population, gap closure was accelerated again (Fig. 4b, c). Notably,
HC-cSCCs showed faster gap closure than RDEB-cSCC and primary $\mathrm{HC}-\mathrm{KC}$, the latter two showing a similar migratory potential (Fig. 4d, e). When overexpressing miR$10 \mathrm{~b}$ in $\mathrm{HC}-\mathrm{KCs}$, no impact on migration was observed (data not shown). The reason for the unexpected delay in gap closure in RDEB-cSCCs remains speculative, but might be due to prior exposure of RDEB-cells to pathological processes like chronic inflammation, or the difference in matrix composition, which is dramatically altered in RDEB patients due to C7 absence [5-9]. Still, our results show that RDEBSCCs migrate more slowly that HC-cSCCs in general, and miR-10b has an impact on mobility. No impact on proliferation was observed in the presence or absence of miR-10b.

Taken together, our data suggest for the first time that CSC-like properties can be conferred by miR-10b, a heretofore unknown aspect of miR-10b driven malignancy in cSCCs, and indicates an impact on motility in RDEB.

\section{DIAPH2 is deregulated in RDEB-CSCC and predicted to be a target of miR-10b}

To analyze the impact of miR-10b on its targetome, we first screened the scientific literature in an automated text-mining approach for miR-10b in cancer, which highlighted the previously reported, direct miR-10b target transcription factor HOXD10 [14], (Supplementary Fig. S7A in Additional File 1). We observed significantly reduced levels of HOXD10 protein in three RDEB-CSCC cell lines compared to $\mathrm{HC}-\mathrm{KC}$, and also in an RDEBcSCC tissue section, compared to HC- and RDEB-skin [34], (Supplementary Fig. S7B-D in Additional File 1).

To next identify novel downstream targets of miR-10b, data driven miR-10b target identification was implemented based on transcriptome data generated from miRNA microarray-matched RDEB-cSCC and RDEB-KC samples. Of the 576 differentially expressed genes identified ( $\geq 2$-fold $\downarrow \uparrow)$ in RDEB-CSCCs, 114 were reported in merged repository data $(n=3923)$ of validated miR-10 targets (miRTarbase v6.1), as well as computationally predicted targets by seed sequence and evolutionary conservation (TargetScan v7.2). Dysregulated putative miR-10 interaction partners were further prioritized by strength of their inverse correlation of expression with miR-10 signal (Supplementary Fig. S7E in Additional File 1). To nominate a disease relevant miR-10b target, we analyzed publicly available survival data from metastatic stage IV head and neck squamous cell carcinoma (HNSCC) ( $n=86$ patients, The Cancer Genome Atlas / TCGA), as this cancer type was previously described to have high genetic similarities to RDEB-cSCC [8]. The top 20 candidate miR-10b targets with highest inverse correlation were then used to stratify HNSCC patients (Supplementary Fig. S8A and Supplementary Table S6 in Additional File 1). We nominated diaphanous related formin 2 (DIAPH2) for further evaluation based on significant differences in Kaplan Meier survival curves $(p<0.05$, log- 


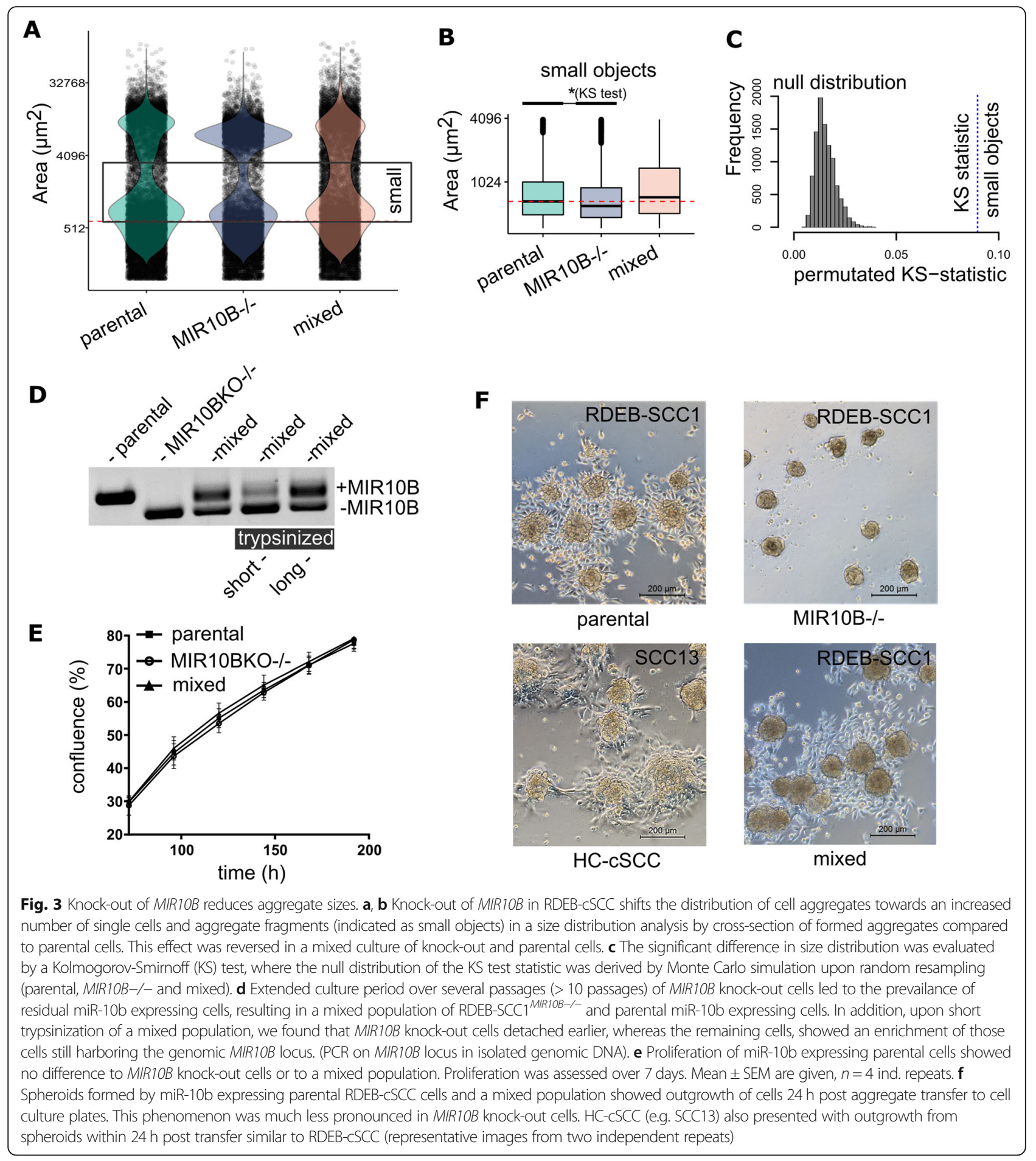

rank test, Fig. 5a), together with the fact that it was listed as a putative target of miR-10b. Its potential disease-relevance was substantiated by its recent association with colon carcinoma, and its potential role in actin-organization and microtubule stabilization [35-37]. When re-analyzing normalized RNA-seq data generated by Cho et al., retrieved from GEOrepository (GSE111582), both, DIAPH2 and HOXD10, showed lower expression in RDEB-cSCC versus RDEB-skin tissue [8], (Supplementary Fig. S8B,C in Additional File 1).

To confirm DIAPH2 as a direct target of miR-10b, a dual luciferase reporter assay was established by cloning the 3'UTRs of DIAPH2 and HOXD10 as a control, respectively, downstream of a firefly luciferase reporter gene. Constructs were then co-transfected with a miR- 


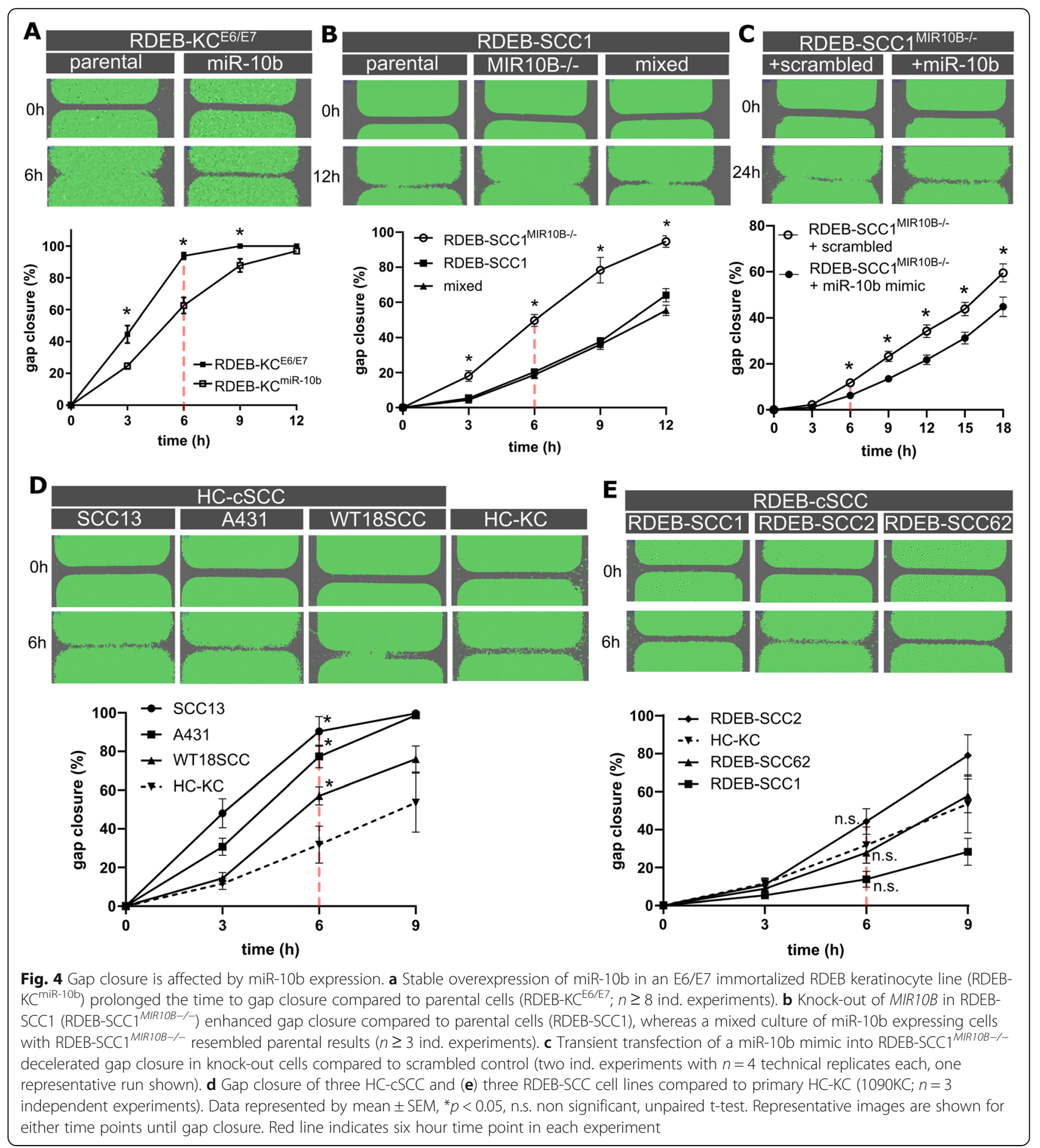

10b mimic into $\mathrm{HC}-\mathrm{KCs}$, which express only low levels of endogenous miR-10b. In the presence of miR-10b mimic, luciferase signal was significantly reduced $(p$ value $^{\text {DIAPH2 }}=0.015 ; p$-value $\left.{ }^{\mathrm{HOXD10}}<0.01\right)$ compared to scrambled (SCR) control (Supplementary Fig. S7F,G in Additional File 1).

We next assessed DIAPH2 expression in cultured cSCCs and control KC. Both, at the mRNA and the protein level,
DIAPH2 was downregulated in RDEB-cSCC cells, with no significant difference between HC- and RDEB-KC (Fig. 5b, d, Supplementary Fig. S7I in Additional File 1). In addition, $D I A P H 2$ was also slightly downregulated in RDEB$\mathrm{KC}^{\text {miR-10b }}$, as shown by sqRT-PCR (Supplementary Fig. S5B in Additional File 1). In HC-cSCCs, DIAPH2 expression levels were not as consistently reduced as in RDEB-cSCC and differed significantly between cell lines (Fig. $5 \mathrm{c}$, d). 

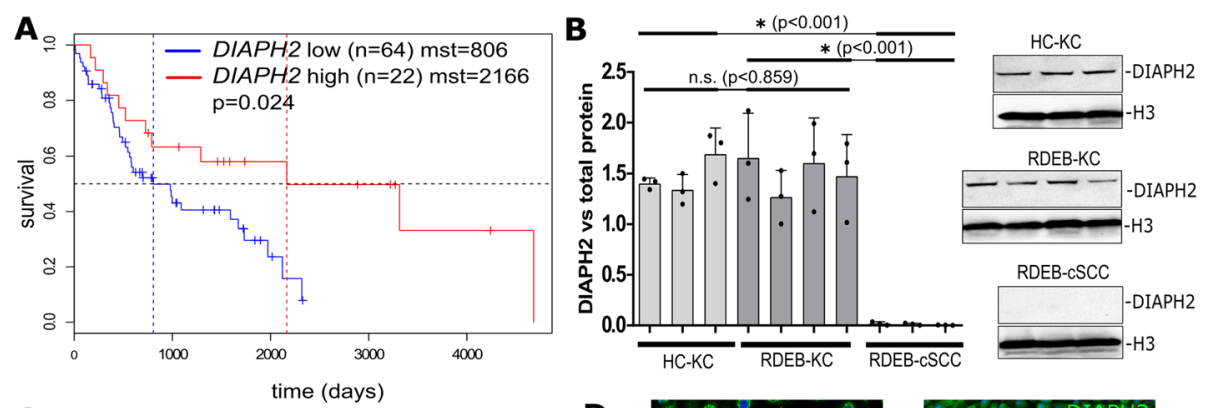

C

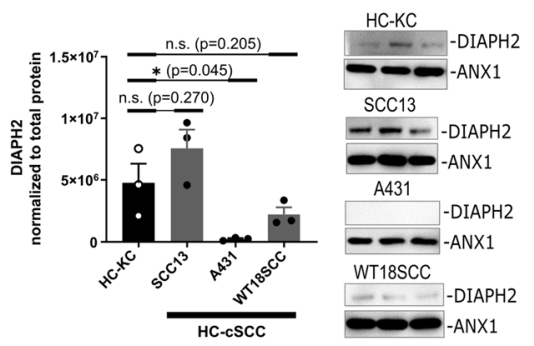

E

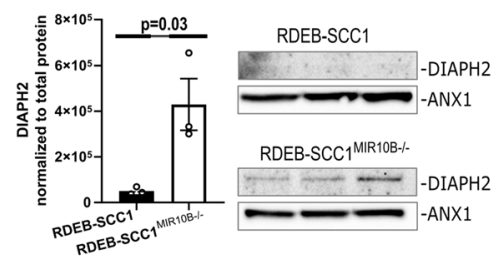

$\mathrm{HC}-\mathrm{KC}$
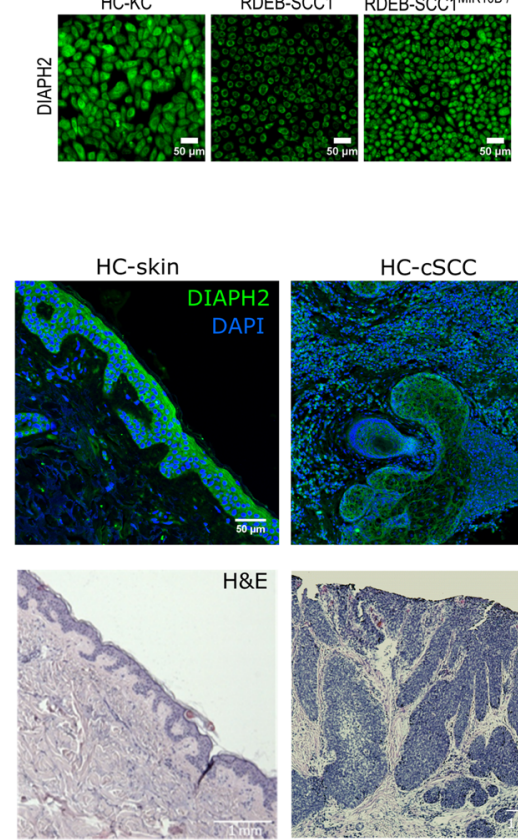

D
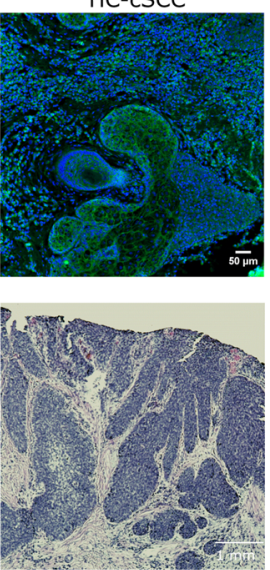
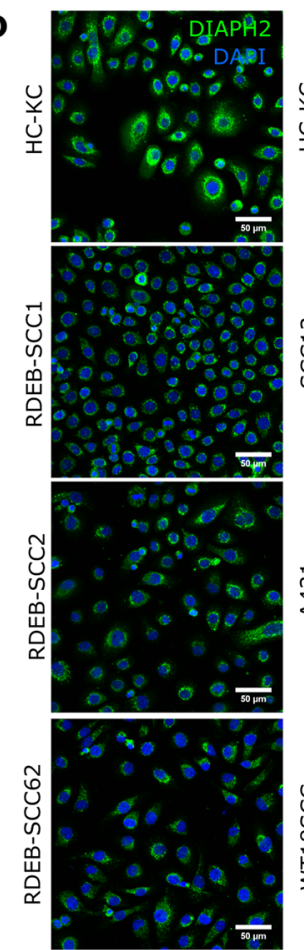

RDEB-skin

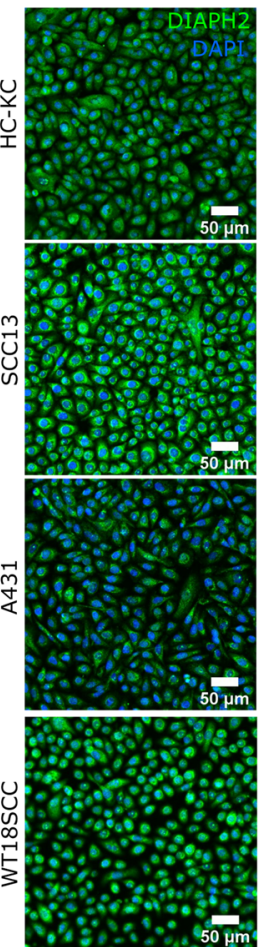

RDEB-cSCC
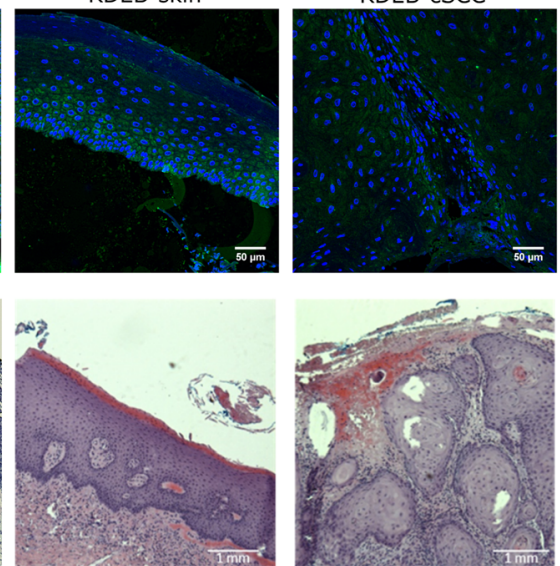

Fig. 5 (See legend on next page.) 


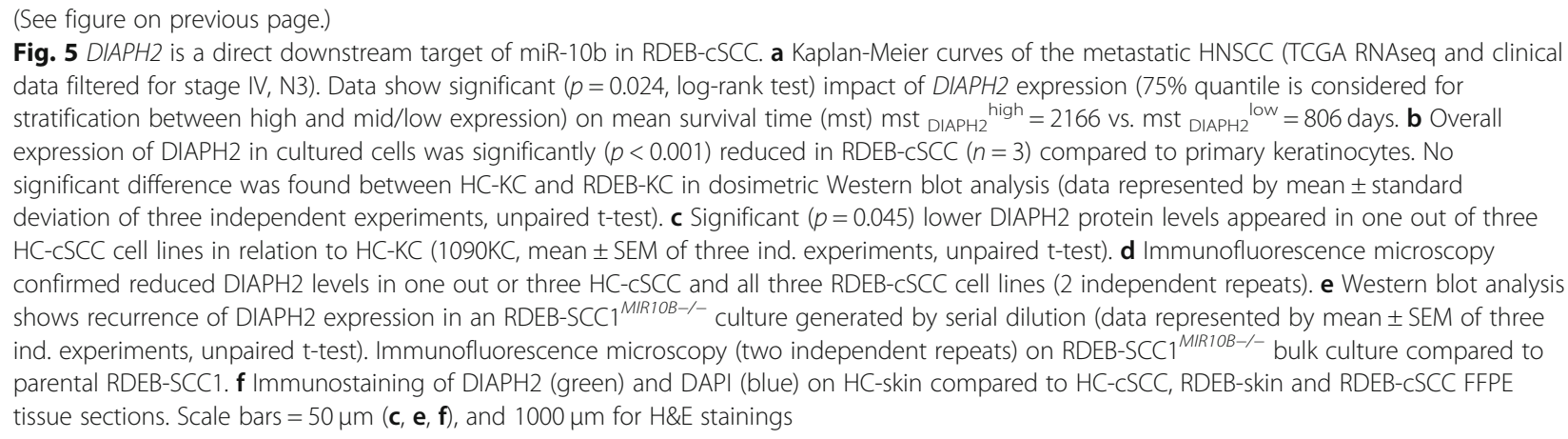

Notably, following transient transfection of HC-KCs with miR-10b mimic, DIAPH2 protein levels were found to be significantly reduced $(\sim 29 \%, p=0.05)$ compared to SCR control (Supplementary Fig. S7H in Additional File 1), and it recurred in RDEB-SCC1 $1^{\text {MIRIOB-/- }}$ as investigated by Western blot analysis and immunofluorescence microscopy (Fig. 5e). DIAPH2 downregulation was also evident in RDEB-cSCC tissue sections (Fig. 5f). Taken together, DIAPH2 appeared as target of miR-10b in RDEB-cSCCs, although inconsistent results in $\mathrm{HC}$-cSCCs indicate further, miR-10b independent regulatory mechnisms.

To examine if a loss of DIAPH2 in keratinocytes phenocopies the observed migratory behavior in RDEB-KC ${ }^{\text {miR-10 }}$, we knocked out DIAPH2 in immortalized HC-KCs using the CRISPR/Cas9 technology ( $\mathrm{HC}-\mathrm{KC}^{\text {DIAPH2-/- }}$ ). Clones were generated by minimal dilution and knock-out was confirmed by Sanger sequencing and Western blot analysis (Supplementary Fig. S5C,D in Additional File 1). While proliferation was not altered, DIAPH2-/- cells showed significantly impaired motility over parental HC-KCs (Supplementary Fig. S5E,F). In addition, when analyzing HC$\mathrm{KC}^{\text {DIAPH2-/- }}$ in a 3D-sphere formation assay, a trend towards enhanced aggregation was observed, with a distribution of spheroid size similar to cSCCs (Supplementary Fig. S5G,H). Overall, the functional impact of DIAPH2 knock-out resembled our observations in RDEB-KC ${ }^{\text {miR-10 }}$ and RDEB-SCC1 ${ }^{\text {MIR1OB-/- }}$.

Our work taken as a whole has demonstrated that miR-10b is overexpressed in cSCC cells and tissues, and that miR-10b confers tumor-associated properties to KCs. In addition, deregulation of DIAPH2, a novel putative miR-10b downstream target, might represent a contributing factor in CSCC malignancy.

\section{Discussion}

Various factors are presumed to contribute to the development of cSCCs in RDEB patients, however, the role of miRNAs in the context of RDEB has not yet been addressed. In this study, our aim was to identify miRNAs involved in the development or progression of particularly aggressive cSCCs in general, and in RDEB-cSCCs in particular, by profiling the miRNome of human cSCCs, as well as keratinocytes. As miR-10b was found to be significantly upregulated in CSCCs in vitro and in situ, we next examined its functional impact on cultured human keratinocytes. We conducted 3D-sphere formation assays using keratinocytes overexpressing miR-10b, and cSCC cells where the MIR1OB gene locus had been knocked-out. While overexpression of miR-10b improved the ability of cells to form stable 3D-spheroids, similar to those derived from aggressive cSCC cells, knock-out significantly reduced spheroid stability. As spheroid formation is a capacity for anchorage-independent survival, generally attributed to cells with the potential to initiate tumor growth, such as CSCs, this shift towards stemness upon miR-10b overexpression in RDEB-keratinocytes was confirmed through an enrichment of $\mathrm{CD} 44^{\text {high }} / \mathrm{CD} 24^{-/ \text {low }}$ cells [38]. Unfortunately, tumor initiation experiments in immunodeficient mice did not result in the engraftment of cells, or the development of a tumor, likely due to their overall nonmalignant background of RDEB keratinocytes. Notably, not all RDEB-cSCC lines are able to successfully engraft in immunodeficient mice, highlighting that other factors, such as a supportive microenvironment, are required. However, the observation that miR-10b is overexpressed and may have a functional role in mediating stemness highlights a novel aspect of miR-10b function in $\mathrm{CSCC}$, that is separate from its well demonstrated role in EMT.

As increased motility is considered as a hallmark of tumor cells undergoing EMT, we were interested in the effect of miR-10b on KCs and cSCCs. When next performing migration assays using miR-10b overexpressing and knock-out cells, we found an attenuated gap closure in the presence of miR-10b, which we also observed when reintroducing miR$10 \mathrm{~b}$ in knock-out cells. Also RDEB-cSCC cell lines showed no enhanced gap closure compared to keratinocytes, which was contrary to our expectation that cancer cells migrate faster than non-malignant controls. However, opposed to RDEB-cSCCs, HC-cSCCs showed an increased migratory behavior over control cells. This leads to the assumption, that while there are clear similarities between HC-cSCCs and RDEB-CSCC in miR-10b expression levels and spheroid 
formation capacities, and miR-10b confers tumor spheroid formation capacities, there are also major differences between the two different types of cSCCs, that affect their migratory behavior. Obviously, the absence of the ECM protein type-VII collagen is a major contributor to tumor formation in RDEB [6-9]. Loss of COL7A1 leads to impaired re-epithelialization through perturbation of laminin-332 organization and abrogation of strictly polarized expression of integrin $\alpha 6 \beta 4$ in basal keratinocytes, which together finally impacts guided keratinocyte migration - an essential step in wound closure [39].

Overall, it has been repeatedly reported that EMT is necessary for cells to disseminate from the primary tumor and become circulating tumor cells (CTCs), complementary models of metastasis exist [31, 40]. One pre-requisite for CTCs is to maintain their ability to revert from the mesenchymal to the epithelial phenotype (MET). Another is based on the concept of "collective" or "cohort" migration. In this case it is assumed that epithelial-like and mesenchymal-like cancer cells circulate in clusters and act cooperatively to promote metastatic growth, without the need to undergo complete EMT/MET [41]. Ultimately, a key event during metastasis is that cells with a tumorinitiating capacity reach and establish at distant sites to form new colonies, which requires cell adhesion [31]. We hypothesize that the increased adhesive potential and sphere formation capacity following overexpression of miR$10 \mathrm{~b}$, might not only increase cell survival in the circulation, but also facilitate extravasation at new sites, and explains in part the propensity of aggressive cSCCs to metastasize [42].

Alternatively, changes in the actin- and / or microtubule cytoskeleton, which impact cell motility [43], could account for slower wound closure rates and increased stickiness in RDEB skin. In this context, the putative miR-10b target DIAPH2, a protein belonging to the formin homology family, which has previously been associated with actin filament assembly, microtubule formation and vesicle shuttling, might be a modulator of cell motility in RDEB-cSCCs [44, 45]. In this study, DIAPH2 was predicted to be a target of miR-10b in silico, which we additionally confirmed using a luciferase-reporter assays. Notably, while miR-10b overexpression did not result in efficient knock-down of DIAPH2 in RDEB-KCs, a significant downregulation of DIAPH2 was observed in RDEBCSCC expressing high endogenous levels of miR-10b, and re-expression of DIAPH2 was demonstrated upon MIR10B knock-out in RDEB-cSCC cells. This indicates potential alternative pathways of DIAPH2 regulation in non-malignant cells. Nonetheless, CRISPR-mediated knock-out of DIAPH2 resulted in functional outcomes similar to those observed in miR-10b overexpressing keratinocytes, including attenuated migration and an enhanced capacity to form 3D-spheroids. While only limited information on DIAPH2 is available, DIAPH3, a closely related family member with $57 \%$ sequence homology and several common protein binding domains, does promote cell growth and metastasis in hepatocellular carcinoma [46]. Supporting our findings, knock-down of DIAPH3 results in reduced migration [47]. DIAPH2 is predicted to interact with small RHO-GTPases, which are implicated in motility, and frequently downregulated in different types of SCCs $[48,49]$. In line with this, our microarray data also indicate a slight downregulation of $R H O C$ and $R H O D$ in RDEB-cSCC. Considering this, a similar function of DIAPH2 in the context of cSCCs is likely but needs to be further explored.

In summary, overexpression of miR-10b impacts cellular processes at various levels. The extent of the response is dependent on the cellular context, the presence of certain target mRNAs, and/or mutations in those target mRNAs [50]. In the context of cSCCs, we showed that miR-10b confers anchorage-independent spheroid formation capacities, indicating a phenotypic shift towards stem cell-like properties. We hypothesize, that these findings might be involved in tumor progression at the stages of extravasation and metastatic colonization, processes in which both anchorage-free survival, as well as cell surface interaction, are important pre-requisites.

\section{Conclusion}

Overall, our results demonstrate for the first time the upregulation of miR-10b in aggressive cSCCs. While new, these findings provide a potential so far unreported prometastatic function of miR-10b, as this miRNA confers cancer stem cell-like properties, like increased adhesion, spheroid formation capacities and associated cellular outgrowth, which are essential characteristics associated with metastasis. However, also differences between RDEBcSCC and HC-cSCC cells were observed, affecting their migratory potential, which point towards a potential impact of the respective genetic background. The results of this study provide an exciting new opportunity for the development of future therapies to treat this rare and debilitating disease. In addition, miR-10b expression in cSCC might hold potential as a biomarker for early diagnosis and / or therapy monitoring in the context of aggressive CSCC, as these tumors often escape early detection due to their predominant emergence within chronic wounds.

\section{Supplementary information}

Supplementary information accompanies this paper at https://doi.org/10. 1186/s12964-020-00550-9.

Additional file 1. Supplementary information and data: Additional information on cell lines, bioinformatic data processing, CRISPR/Cas9mediated knock-out of DIAPH2, flow cytometry for CD44 and CD24 expression, miRNA library preparation and next generation sequencing, Luciferase-reporter assay. Supplementary data summarized in tables: cell lines, antibodies, primers sequences, top up- and down-regulated miRNAs 
in microarray analysis: RDEB-CSCC versus RDEB-KCs, and $\mathrm{HC}-\mathrm{CSCC}$ versus HC-KC, miR-10b target selection and validation. Supplementary experimental data in figures: miR-10b target selection and validation; controls for miRNA probe specificity in in situ hybridization on FFPE tissue sections: miRNA probe specificity for in situ hybridization on cultured cells and single cell resolution image-based data analysis workflow; miRNA probe specificity for in situ hybridization on cultured cells; generation of experimental cell lines; characterization of experimental cell lines; validation of miR-10b targets HOXD10 and DIAPH2; survival analysis and target evaluation.

\section{Abbreviations}

CSCC: cutaneous squamous cell carcinomas; CSCs: Cancer stem cells; CTCs: Circulating tumor cells; DIAPH2: Diaphanous Related Formin 2; ECM: Extracellular matrix; EMT: Epithelial to mesenchymal transition; FDR: False discovery rate; FFPE: Formalin-fixed paraffin embedded; GAPDH: Glycerinaldehyd-3-phosphat-dehydrogenase; H\&E: Hematoxilin \& eosin; HC: Healthy control; HNSCC: Head and neck squamous cell carcinoma; HOXD: Homeobox D; IHC: Immunohistochemistry; KC: Keratinocytes; LNA: Locked-nucleid acid; MET: Mesenchymal to epithelial transition; miRNAs: micro-RNAs; onco-miRs: oncogenic miRNAs; PCA: Principal component analysis; RDEB: Recessive-dystrophic epidermolysis bullosa; LM: Lymphnode metastasis; RNA-seq: RNA sequencing; SCC: Squamous cell carcinomas; SCR: Scrambled; SEM: Standard error of mean; SSC: Salinesodium citrate; TCGA: The Cancer Genome Atlas; TUBA1: Tubulin alpha 1

\section{Acknowledgements}

We would like to thank Prof. Andrew South and Prof. Leena BrucknerTuderman for providing cell lines RDEB-SCC1 and RDEB-SCC2, and Dr. Tzipi Cohen Hyams and A/Prof Murray Killingsworth, Imaging Facility of the Ingham Institute for Applied Medical Research, Sydney for their technical support. In addition we would like to thank the group of Prof. Herbert Reitsamer, University Clinic of Ophthalmology and Optometry / Paracelsus Medical University Salzburg, for providing access to their confocal laserscanning unit (Axio Observer Z1 attached to LSM710, Zeiss). Finally, Andrea Wagner from the Institute of Tendon \& Bone Regeneration, Spinal Cord Injury and Tissue Regeneration Center Salzburg (SCI-TReCS), Paracelsus Medical University, for her support in IHC-tissue processing.

\section{Authors' contributions}

MW and RZ performed experiments, designed and generated data processing workflows, interpreted the experimental results and assisted in conception and writing of the manuscript. MA participated in the GRT-PCR, immunostaining, western blots, FACS and assisted in data interpretation. JPH participated in FACS analysis and assisted in manuscript writing. CGG, MR, SH, EMM, DS, TK participated in isolating primary cells, cloning of retroviral vectors and generation of cell lines. NN and JP performed miRNA sequencing. MS, PDS and ASM assisted in the optimization of the miRNA ISH protocol. TL, JWB, JR and ASM contributed in writing and reviewing the manuscript. WW conceived and designed this study, interpreted the data and wrote the manuscript. All authors reviewed the manuscript and approved the submission.

\section{Funding}

This project was supported by the Austrian Science Fund (FWF): P29343-B30, The Paracelsus Medical University Salzburg (A-16/02/025-WAL), DEBRA Austria and DEBRA Australia.

\section{Availability of data and materials}

The data that support the findings of this study were submitted to the Gene Expression Omnibus Database (Accession: GSE130767 for miRNA and GSE130925 for transcriptome microarray data). And the data are available from https://www.ncbi.nlm.nih.gov/geo/query/acc.cgi?acc=GSE130767 and https://www.ncbi.nlm.nih.gov/geo/query/acc.cgi?acc=GSE130925.

\section{Ethics approval and consent to participate}

RDEB tissue samples collected in Salzburg were obtained from patients undergoing dermatological surgery upon written, informed consent. Ethical approval was granted by the ethics committee of the county of Salzburg (vote number: 415-EP/73/192-2013) for RDEB and 1090KC cell isolations. For the generation of "SKC"-HC cell lines human full-thickness skin was obtained from biologic waste material during plastic reconstructive surgery after informed consent as approved by the ethical committee of the county of Salzburg (vote number: 415-E/1990/8-216). Skin was processed as described previously [51].

\section{Consent for publication}

Not applicable.

\section{Competing interests}

The authors declare that they have no competing interests.

\section{Author details}

'EB House Austria, Research Program for Molecular Therapy of Genodermatoses, Department of Dermatology \& Allergology, University Hospital of the Paracelsus Medical University, 5020 Salzburg, Austria. ${ }^{2}$ Red Cross Transfusion Service of Upper Austria, 4020 Linz, Austria. ${ }^{3}$ Center for Medical Research, Medical Faculty, Johannes-Kepler-University, 4020 Linz, Austria. ${ }^{4}$ Medical Oncology, Ingham Institute for Applied Medical Research, Liverpool, NSW 2170, Australia. ${ }^{5}$ School of Medicine, Western Sydney University, Campbelltown, NSW 2560, Australia. ${ }^{6}$ Department of Dermatology, University Hospital of the Paracelsus Medical University Salzburg, 5020 Salzburg, Austria. ${ }^{7}$ Institute of Experimental \& Clinical Cell Therapy, Paracelsus Medical University, 5020 Salzburg, Austria. ${ }^{8}$ School of Medical Science, University of New South Wales, Kensington, NSW 2052, Australia.

Received: 30 June 2019 Accepted: 11 March 2020

Published online: 10 April 2020

\section{References}

1. Que SKT, Zwald FO, Schmults CD. Cutaneous squamous cell carcinoma: incidence, risk factors, diagnosis, and staging. J Am Acad Dermatol. 2018; 78(2):237-47.

2. Fine JD, Bruckner-Tuderman L, Eady RAJ, Bauer EA, Bauer JW, Has C, et al. Inherited epidermolysis bullosa: updated recommendations on diagnosis and classification. J Am Acad Dermatol. 2014;70(6):1103-26.

3. van den Akker PC, Jonkman MF, Rengaw T, Bruckner-Tuderman L, Has C, Bauer JW, et al. The international dystrophic epidermolysis bullosa patient registry: an online database of dystrophic epidermolysis bullosa patients and their COL7A1 mutations. Hum Mutat. 2011;32(10):1100-7.

4. Fine J-D, Johnson LB, Weiner M, Li K-P, Suchindran C. Epidermolysis bullosa and the risk of life-threatening cancers: the national EB registry experience, 1986-2006. J Am Acad Dermatol. 2009;60(2):203-11.

5. Knaup J, Gruber C, Krammer B, Ziegler V, Bauer J, Verwanger T. TGFbetasignaling in squamous cell carcinoma occurring in recessive dystrophic epidermolysis bullosa. Anal Cell Pathol. 2011;34(6):339-53.

6. Nyström A, Thriene K, Mittapalli V, Kern JS, Kiritsi D, Dengjel J, et al. Losartan ameliorates dystrophic epidermolysis bullosa and uncovers new disease mechanisms. EMBO Mol Med. 2015;7(9):1211-28.

7. Martins VL, Caley MP, Moore K, Szentpetery Z, Marsh ST, Murrell DF, et al. Suppression of TGFbeta and Angiogenesis by Type VII Collagen in Cutaneous SCC. J Natl Cancer Inst. 2016;108(1):djv293.

8. Cho RJ, Alexandrov LB, den Breems NY, Atanasova VS, Farshchian M,

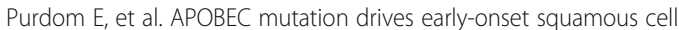
carcinomas in recessive dystrophic epidermolysis bullosa. Sci Transl Med. 2018;10(455):eaas9668.

9. Mittapalli VR, Madl J, Löffek S, Kiritsi D, Kern JS, Römer W, et al. Injury-driven stiffening of the dermis expedites skin carcinoma progression. Cancer Res. 2015;76(4):940-52.

10. Bartel DP. MicroRNA target recognition and regulatory functions. Cell. 2009; 136(2):215-33.

11. Rupaimoole R, Han HD, Lopez-Berestein G, Sood AK. MicroRNA therapeutics: principles, expectations, and challenges. Chin J Cancer. 2011;30(6):368-70.

12. Esteller M. Non-coding RNAs in human disease. Nat Rev Genet. 2011;12(12): 861-74.

13. Ma L, Reinhardt F, Pan E, Soutschek J, Bhat B, Marcusson EG, et al. Therapeutic silencing of miR-10b inhibits metastasis in a mouse mammary tumor model. Nat Biotechnol. 2010;28(4):341-7.

14. Ma L, Teruya-Feldstein J, Weinberg RA. Tumour invasion and metastasis initiated by microRNA-10b in breast cancer. Nature. 2007;449(7163):682-8. 
15. Plummer PN, Freeman R, Taft RJ, Vider J, Sax M, Umer BA, et al. MicroRNAs regulate tumor angiogenesis modulated by endothelial progenitor cells. Cancer Res. 2013;73(1):341-52.

16. Fantini S, Salsi V, Zappavigna V. HOX cluster-embedded micro-RNAs and cancer. Biochim Biophys Acta Rev Cancer. 2018;1869(2):230-47.

17. Tehler D, Høyland-Kroghsbo NM, Lund AH. The miR-10 microRNA precursor family. RNA Biol. 2011;8(5):728-34.

18. Gasch C, Plummer PN, Jovanovic L, McInnes LM, Wescott D, Saunders CM, et al. Heterogeneity of miR-10b expression in circulating tumor cells. Sci Rep. 2015;5:15980.

19. Abdelmaksoud-Dammak R, Chamtouri N, Triki M, Saadallah-Kallel A, Ayadi W, Charfi S, et al. Overexpression of miR-10b in colorectal cancer patients: correlation with TWIST-1 and E-cadherin expression. Tumour Biol. 2017;39(3): 1010428317695916.

20. Obermannova R, Redova-Lojova M, Vychytilova-Faltejskova P, Grell P, Cho WC, Sachlova M, et al. Tumor expression of miR-10b, miR-21, miR-143 and miR-145 is related to Clinicopathological features of gastric Cancer in a central European population. Anticancer Res. 2018;38(6):3719-24.

21. Bai M, Zhang H, Si L, Yu N, Zeng A, Zhao R. Upregulation of serum miR-10b is associated with poor prognosis in patients with melanoma. J Cancer. 2017;8(13):2487-91.

22. Zhang J, Yang J, Zhang $X, X u$ J, Sun Y, Zhang P. MicroRNA-10b expression in breast cancer and its clinical association. PLoS One. 2018;13(2):e0192509.

23. Gee HE, Camps C, Buffa FM, Colella S, Sheldon H, Gleadle JM, et al. MicroRNA-10b and breast cancer metastasis. Nature. 2008;455(7216):E8-9 author reply $\mathrm{E9}$.

24. Khella HWZ, Daniel N, Youssef L, Scorilas A, Nofech-Mozes R, Mirham L, et al. miR-10b is a prognostic marker in clear cell renal cell carcinoma. J Clin Pathol. 2017;70(10):854-9.

25. Gehwolf R, Wagner A, Lehner C, Bradshaw AD, Scharler C, Niestrawska JA, et al. Pleiotropic roles of the matricellular protein Sparc in tendon maturation and ageing. Sci Rep. 2016;6:32635.

26. Peking P, Breitenbach JS, Ablinger M, Muss WH, Poetschke FJ, Kocher T, et al. An ex vivo RNA trans-splicing strategy to correct human generalized severe epidermolysis bullosa simplex. Br J Dermatol. 2019;180(1):141-8.

27. Mehta A, Baltimore D. MicroRNAs as regulatory elements in immune system logic. Nat Rev Immunol. 2016;16(5):279-94.

28. O'Connell RM, Rao DS, Chaudhuri AA, Baltimore D. Physiological and pathological roles for microRNAs in the immune system. Nat Rev Immunol. 2010;10(2):111-22.

29. Chen L, Al-Mossawi MH, Ridley A, Sekine T, Hammitzsch A, de Wit J, et al. miR-10b-5p is a novel Th17 regulator present in Th17 cells from ankylosing spondylitis. Ann Rheum Dis. 2017;76(3):620-5.

30. Bourguignon LY, Wong G, Earle C, Krueger K, Spevak CC. Hyaluronan-CD44 interaction promotes c-Src-mediated twist signaling, microRNA-10b expression, and RhoA/RhoC up-regulation, leading to rho-kinase-associated cytoskeleton activation and breast tumor cell invasion. J Biol Chem. 2010; 285(47):36721-35.

31. Lambert AW, Pattabiraman DR, Weinberg RA. Emerging biological principles of metastasis. Cell. 2017;168(4):670-91.

32. Hirschhaeuser F, Menne H, Dittfeld C, West J, Mueller-Klieser W, KunzSchughart LA. Multicellular tumor spheroids: an underestimated tool is catching up again. J Biotechnol. 2010;148(1):3-15.

33. Li W, Ma H, Zhang J, Zhu L, Wang C, Yang Y. Unraveling the roles of CD44/ CD24 and ALDH1 as cancer stem cell markers in tumorigenesis and metastasis. Sci Rep. 2017;7(1):13856.

34. Xiao H, Li H, Yu G, Xiao W, Hu J, Tang K, et al. MicroRNA-10b promotes migration and invasion through KLF4 and HOXD10 in human bladder cancer. Oncol Rep. 2014;31(4):1832-8.

35. Lin YN, Windhorst S. Diaphanous-related formin 1 as a target for tumor therapy. Biochem Soc Trans. 2016;44(5):1289-93.

36. Morley S, You S, Pollan S, Choi J, Zhou B, Hager MH, et al. Regulation of microtubule dynamics by DIAPH3 influences amoeboid tumor cell mechanics and sensitivity to taxanes. Sci Rep. 2015;5:12136.

37. Grueb SS, Muhs S, Popp Y, Schmitt S, Geyer M, Lin YN, et al. The formin Drosophila homologue of Diaphanous2 (Diaph2) controls microtubule dynamics in colorectal cancer cells independent of its FH2-domain. Sci Rep. 2019;9(1):5352

38. Bao B, Ahmad A, Azmi AS, Ali S, Sarkar FH. Overview of cancer stem cells (CSCs) and mechanisms of their regulation: implications for cancer therapy. Curr Protoc Pharmacol. 2013;14:14-25.
39. Nyström A, Velati D, Mittapalli VR, Fritsch A, Kern JS, Bruckner-Tuderman L. Collageen VII plays a dual role in wound healing. J Clin Invest. 2013;123(8):3498-509.

40. Thiery JP, Acloque H, Huang RYJ, Nieto MA. Epithelial-Mesenchymal Transitions in Development and Disease. Cell. 2009:139:871-90.

41. Friedl P, Gilmour D. Collective cell migration in morphogenesis, regeneration and cancer. Nat Rev Mol Cell Biol. 2009;10(7):445-57.

42. Liu X, Taftaf R, Kawaguchi M, Chang YF, Chen W, Entenberg D, et al. Homophilic CD44 interactions mediate tumor cell aggregation and polyclonal metastasis in patient-derived breast Cancer models. Cancer Discov. 2019;9(1):96-113.

43. Tang DD, Gerlach BD. The roles and regulation of the actin cytoskeleton, intermediate filaments and microtubules in smooth muscle cell migration. Respir Res. 2017;18(1):54.

44. Goode BL, Eck MJ. Mechanism and function of formins in the control of actin assembly. Annu Rev Biochem. 2007;76:593-627.

45. Hager MH, Morley S, Bielenberg DR, Gao S, Morello M, Holcomb IN, et al. DIAPH3 governs the cellular transition to the amoeboid tumour phenotype. EMBO Mol Med. 2012;4(8):743-60.

46. Katoh M, Katoh M. Identification and characterization of human DIAPH3 gene in silico. Int J Mol Med. 2004;13(3):473-8.

47. Dong L, Li Z, Xue L, Li G, Zhang C, Cai Z, et al. DIAPH3 promoted the growth, migration and metastasis of hepatocellular carcinoma cells by activating betacatenin/TCF signaling. Mol Cell Biochem. 2018;438(1-2):183-90.

48. Jiang L, Liu X, Kolokythas A, Yu J, Wang A, Heidbreder CE, et al. Downregulation of the rho GTPase signaling pathway is involved in the microRNA-138-mediated inhibition of cell migration and invasion in tongue squamous cell carcinoma. Int J Cancer. 2010;127(3):505-12.

49. Wang H, Wang Y, Liang B, He F, Li Y, Che J, et al. The Rho GTPase RhoE exerts tumor-suppressing effects in human esophageal squamous cell carcinoma via negatively regulating epidermal growth factor receptor. J Cancer Res Ther. 2016;12(Supplement):60-3.

50. Breitenbach JS, Rinnerthaler M, Trost A, Weber M, Klausegger A, Gruber C, et al. Transcriptome and ultrastructural changes in dystrophic Epidermolysis bullosa resemble skin aging. Aging (Albany NY). 2015;7(6):389-411.

51. Hochmann S, Mittermeir M, Santic R, Koszik F, Griessner L, Sonderegger AS, et al. Evaluation of modified interferon alpha mRNA constructs for the treatment of non-melanoma skin cancer. Sci Rep. 2018;8(1):12954.

\section{Publisher's Note}

Springer Nature remains neutral with regard to jurisdictional claims in published maps and institutional affiliations.

Ready to submit your research? Choose BMC and benefit from:

- fast, convenient online submission

- thorough peer review by experienced researchers in your field

- rapid publication on acceptance

- support for research data, including large and complex data types

- gold Open Access which fosters wider collaboration and increased citations

- maximum visibility for your research: over $100 \mathrm{M}$ website views per year

At $\mathrm{BMC}$, research is always in progress.

Learn more biomedcentral.com/submissions 